\title{
Direct Retinal Signals for Virtual Environments
}

\author{
E. Koskinen \\ University of Tampere \\ Faculty of Communication Sciences \\ 33014 Tampere, Finland \\ koskinen.eero@gmail.com
}

\author{
I. Rakkolainen \\ University of Tampere \\ Faculty of Communication Sciences \\ 33014 Tampere, Finland \\ ismo.rakkolainen@uta.fi
}

\author{
R. Raisamo \\ University of Tampere \\ Faculty of Communication Sciences \\ 33014 Tampere, Finland \\ roope.raisamo@uta.fi
}

\begin{abstract}
We present a novel signaling method for head-mounted displays, which surpasses eye (pupil) and delivers guiding light signals directly to retina through tissue near the eyes. This method preserves full visual acuity on the display and does not block view to the scene, while also delivering additional visual signals.
\end{abstract}

\section{CCS CONCEPTS}

- Human-centered computing $\rightarrow$ Displays and imagers $\bullet$ Interface design prototyping $\bullet$ Gestural input $\bullet$ Computing methodologies $\rightarrow$ Mixed / augmented reality $\bullet$ Virtual reality $\bullet$ Hardware $\rightarrow$ Displays and imagers

\section{KEYWORDS}

HMD; VR viewers; virtual reality; $360^{\circ}$ video; $3 \mathrm{D}$ interaction

\section{ACM Reference format:}

E. Koskinen, I. Rakkolainen, and R. Raisamo. 2017. Direct Retinal Signals for Virtual Environments. In Proceedings of ACM VRST conference, Gothenburg, Sweden, November 2017 (VRST'17), 2 pages.

DOI: https://doi.org/10.1145/3139131.3141197

\section{INTRODUCTION}

Head-mounted displays (HMD) are used to view virtual reality $(\mathrm{VR})$, augmented reality (AR) and spherical $360^{\circ}$ (horizontal) $\times$ $180^{\circ}$ (vertical) video $\left(360^{\circ}\right.$ video). VR has recently become feasible and affordable for consumers, as powerful 3D graphics rendering and high-resolution HMDs have become low-cost.

Our contribution in this paper is to demonstrate a visual signaling system, which does not occlude the visual view of a HMD, but can deliver a blurry visual signal directly to retina with limited directionality. It can be retrofitted to any existing HMDs. We first discuss previous HMDs and alternative visual delivery systems, then present our prototype, discuss its limitations and potential improvements, and finally give conclusions.

Augmented information such as signals, arrows or lights can be shown on top of the field-of-view of the HMD, but they then block some of the image and may distract the user. Also lights in

Permission to make digital or hard copies of part or all of this work for personal or classroom use is granted without fee provided that copies are not made or distributed for profit or commercial advantage and that copies bear this notice and the full citation on the first page. Copyrights for third-party components of this work must be honored. For all other uses, contact the Owner/Author.

VRST'17, November 8-10, 2017, Gothenburg, Sweden

(C) 2017 Copyright is held by the owner/author(s).

ACM ISBN 978-1-4503-5548-3/17/11.

https://doi.org/10.1145/3139131.3141197 the peripheral visual field of an HMD without any image have been proposed [e.g., Xiao, Benko 2016] for purposes such as widening the field-of-view or countering the vection effect and thus reducing nausea. Peripheral light with these devices is delivered in a normal manner through the eyes. For purposes such as lucid dreaming, light can be delivered through closed eyes, e.g., iBand+ [Arenar 2017].

Light normally travels to retina through lens and pupil. Alternative non-invasive ways (without surgical implants) to create light perception are to elicit phosphenes transcutaneously in the optic nerve [Evreinova et al. 2010] or transcranially in the visual cortex (occipital lobe) of the brain [Kanai et al. 2008], or as in our novel method to surpass the eye and bring light directly to retina through tissue surrounding the eye (see Fig. 1).

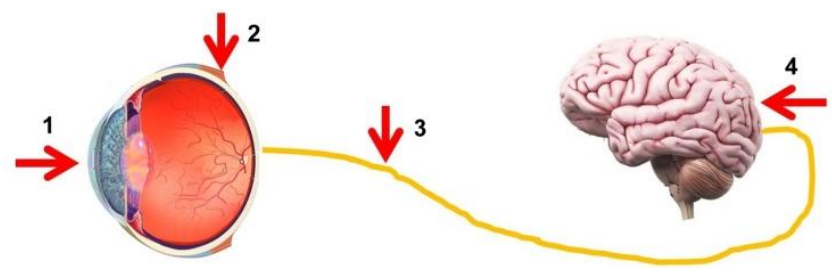

Figure 1: Light is normally delivered through the lens and pupil (1) to the retina. Alternative non-invasive ways are to bring light directly through tissue to the retina (2), or to elicit phosphenes in the optic nerve (3) or in the visual cortex of brain (4).

There are several methods to elicit transient phosphenes. A major problem with many of them is that an input can have unpredictable results between individuals and between various times for the same individual. Stimulation of the optic nerve only gives a brief flash with no spatial information. These alternative methods have been applied to VR or HMDs only in a recent TMS phosphene paper [Losey et al. 2016] and GRS paper [Higuchi et al. 2017]. In our paper we focus on signaling red light through the tissue near eyes, which is a novel idea for HMDs. It is a noninvasive method and can be retrofitted to any HMD.

\section{APPARATUS AND EXPERIMENT}

The gist of the device is a cardboard face mask with an array of 8 red LED lights which is designed to fit over a HTC Vive face mask. The device doesn't block the user's vision over the HMD in any way. The red LEDs are symmetrically mounted on the mask, one LED above and below each eye, and LEDs on the sides of the mask at the eye level and at above it (see Fig. 2). 


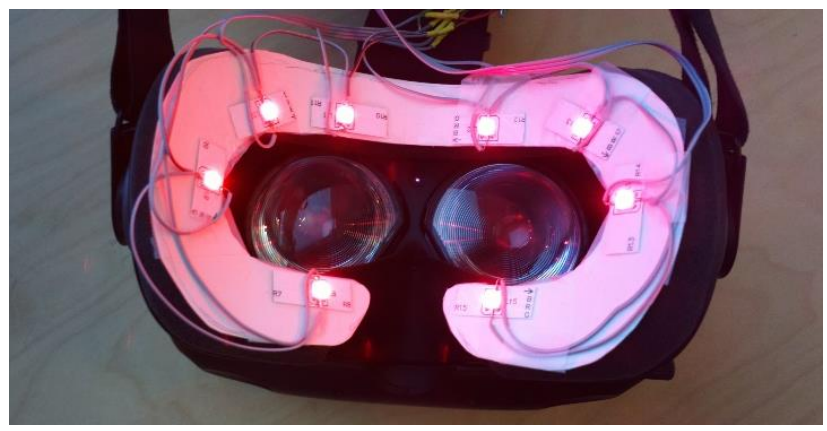

Figure 2: The LED face skin mask is beyond FOV and does not obstruct the HMD view.

The brightness and length of the LED signals are commanded through an Arduino UNO microcontroller with $100 \mathrm{Ohm}$ current limiting resistors. The LEDs are controlled by a simple Arduino program which can control the LEDs individually by setting them on, off or blinking at any given frequency. The device could also be connected to an Arduino Mega microcontroller, which would allow the program to freely alter the brightness of the LEDs. Arduino communicates with Unity over serial port allowing VR applications to (indirectly) control the device.

The user sees a red light dispersed over a large area usually in the peripheral areas of the vision. The perceived light is spatialized to the sides, up or down, but precise direction or clear borders cannot be elaborated. All the light travels through tissue to retina and no light is bleeding onto the interior of the mask.

In order to evaluate the expanded HMD, we compared it with and without the retinal LED feedback in an object searching task. The goal of this initial, informal user test was to get insight in performance, usability and preference for further studies.

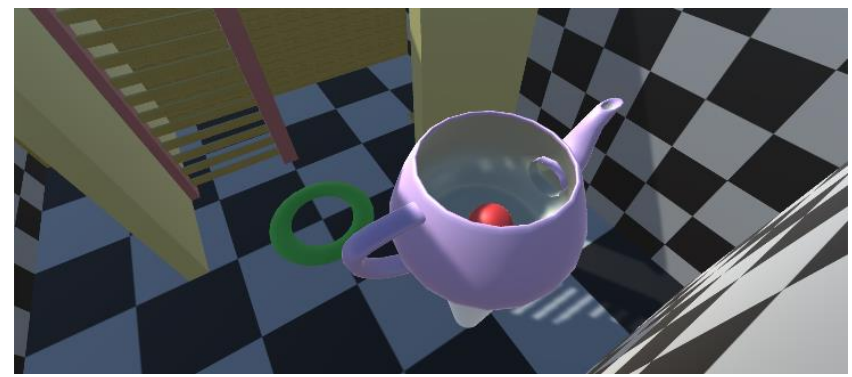

Figure 3: A red ball hidden in a room to be found.

We used a virtual environment of a small room-sized volume where the task was to find objects by looking around. Fig. 3 displays the environment where a red ball is hidden. We used Unity 5.6 to create the environment and HTC Vive HMD to view it. The retinal LED lights guide the user to view to correct direction. The user seeks 10 red balls with and without retinal cues.

We recruited 3 participants (3 male; 24-35 years old, mean age 28) from the laboratory staff and students. They had some previous viewing experience of virtual environments.

\section{RESULTS AND DISCUSSION}

Two participants found the objects faster $(13 \%, 7 \%)$ with the aid of LEDs, but one participant felt during the first few tasks that the LEDs were somewhat confusing, and they slowed him down significantly. Towards the end, he learnt to employ them. In some cases it speeded up the finding significantly. Overall feedback from the participants was very positive. It was stated that the LEDs directly on the left and right side of the eyes helped to turn one's head immediately to the right direction.

The perceived brightness depends very much on the color of the LEDs. Red and yellow light travels and diffuses well through human tissue, but e.g., green or blue light are almost fully attenuated. Change of color, blinking or animating several LEDs, etc. could also be used to deliver some information.

Another important issue is the exact location of the LED lights. Translucent tissue, transparent eyeballs and opaque skull all have an effect on the scattering and blocking of light before it reaches the retina. The perceived light can be very bright in the areas near eye, and dissipates significantly further away. We tested numerous locations around the eyes, and the test indicates that the LEDs should be placed on the skin as near to the eyes as possible without obstructing the HMD view.

Direct retinal lighting could be used also for any other VR glasses and displays such as e.g., CAVEs, if the viewer's head is tracked and she wears a LED facemask.

\section{CONCLUSIONS}

We have created a novel unobtrusive visual signaling system for any HMD or VR viewer. The proof-of-concept prototype can give visual signals without obstructing human FOV and it is also low-cost, light-weight and compact. Our retinal visual signal prototype suggests that it is a promising option for HMDs. We will continue to develop the hardware and software to test related issues further with better prototypes and larger test user sets.

\section{ACKNOWLEDGMENTS}

We thank all the colleagues and reviewers who provided helpful comments on this paper. This work is partially funded by Tekes project \#1444/31/2016 and by Alfred Kordelin Foundation.

\section{REFERENCES}

Arenar B.V. 2017. iBand+ headband. http://www.ibandplus.com/. Accessed on September 22, 2017

Tatiana G. Evreinova, Grigori Evreinov, and Roope Raisamo. 2010. Camera Based Target Acquisition Augmented with Phosphene Sensations. In ICCHP 2010, Part II, K. Miesenberger et al. (Eds.). Lecture Notes in Computer Science, Vol. 6180. Springer-Verlag Berlin Heidelberg, 282-289.

Daiki Higuchi, Kazuma Aoyama, Masahiro Furukawa, Taro Maeda, and Hideyuki Ando. 2017. Position Shift of Phosphene and Attention Attraction in Arbitrary Direction with Galvanic Retina Stimulation. In Proceedings of the 8th Augmented Human International Conference (AH'17), article No. 10.

Ryota Kanai, Leila Chaieb, Andrea Antal, Vincent Walsh, and Walter Paulus. 2008. Frequency-Dependent Electrical Stimulation of the Visual Cortex. Current Biology 18, 23, 1839-1843.

Darby Losey, Andrea Stocco, Justin Abernethy, and Rajesh Rao. 2016. Navigating a 2D Virtual World Using Direct Brain Stimulation. Front. Robot. AI 3, 72.

R. Xiao, and H. Benko. 2016. Augmenting the Field-of-View of Head-Mounted Displays with Sparse Peripheral Displays. ACM CHI 2016, pp. 1221-1232. 